\title{
Lattice QCD calculations of nucleon transverse momentum-dependent parton distributions using clover and domain wall fermions
}

\author{
B. Yoon ${ }^{* a \dagger}$, T. Bhattacharya ${ }^{a}$, M. Engelhardt ${ }^{b}$, J. Green $^{c}$, R. Gupta ${ }^{a}$, P. Hägler ${ }^{d}$, \\ B. Musch ${ }^{d}$, J. Negele ${ }^{e}$, A. Pochinsky ${ }^{e}$, S. Syritsyn ${ }^{f}$
}

${ }^{a}$ Los Alamos National Laboratory, MS B283, P.O. Box 1663, Los Alamos, NM 87545, USA

${ }^{b}$ Department of Physics, New Mexico State University, Las Cruces, NM 88003-8001, USA

${ }^{c}$ Institut für Kernphysik, Johannes Gutenberg-Universität Mainz, 55099 Mainz, Germany

${ }^{d}$ Institut für Theoretische Physik, Universität Regensburg, 93040 Regensburg, Germany

${ }^{e}$ Center for Theoretical Physics, Massachusetts Institute of Technology, Cambridge, MA 02139,

USA

${ }^{f}$ Theory Center, Thomas Jefferson National Accelerator Facility, Newport News, VA 23606, USA

${ }^{\dagger}$ E-mail: boramelanl.gov

\begin{abstract}
We present a lattice QCD calculation of transverse momentum dependent parton distribution functions (TMDs) of protons using staple-shaped Wilson lines. For time-reversal odd observables, we calculate the generalized Sivers and Boer-Mulders transverse momentum shifts in SIDIS and DY cases, and for T-even observables we calculate the transversity related to the tensor charge and the generalized worm-gear shift. The calculation is done on two different $n_{f}=2+1$ ensembles: domain-wall fermion (DWF) with lattice spacing $0.084 \mathrm{fm}$ and pion mass of $297 \mathrm{MeV}$, and clover fermion with lattice spacing $0.114 \mathrm{fm}$ and pion mass of $317 \mathrm{MeV}$. The results from those two different discretizations are consistent with each other.
\end{abstract}

The 33rd International Symposium on Lattice Field Theory,

14 - 18 July 2015

Kobe International Conference Center, Kobe, Japan

${ }^{*}$ Speaker. 


\section{Introduction and Methodology}

Intrinsic motion of partons inside nucleons gives an important picture of the nucleon structure. The intrinsic motion of partons in the transverse plane in momentum space is described by the transverse momentum-dependent parton distributions (TMDs). TMDs have been studied actively both theoretically, i.e., by using the QCD factorization $[1,2,3]$, and experimentally, i.e., COMPASS, HERMES and JLab experiments [4, 5, 6]. Here we report a lattice QCD calculation of the TMDs.

The methodology we use for the calculation of the TMDs is elaborately described in the previous papers [7, 8]. It can be summarized as follows. Considering off-light-cone kinematics from the beginning, TMD correlators are parametrized in terms of the Lorentz-invariant amplitudes, so that they can be calculated on the lattice. Only the ratios of isovector $(u-d)$ TMD observables, that cancel soft factors, multiplicative renormalization factors and disconnected quark loop contributions, are calculated. The Semi-Inclusive Deep-Inelastic Scattering (SIDIS) and Drell-Yan (DY) scattering processes are described by the staple-shape gauge links connecting two separated quarks in the TMD correlators, whose direction and extent are denoted by $v$ and $\eta$, respectively. Here the staple direction $v$ is taken to be space-like in order to avoid the rapidity divergences [9]; light-cone can be approached in the limit of the Collins-Soper parameter $\hat{\zeta} \rightarrow \infty$, guided by a perturbative evolution [10,11].

In this study, we analyze two ensembles; we call them the Clover and DWF ensembles. Lattice parameters are given in Table 1. Unlike the previous study Ref. [8], we use unitary combination of sea and valence quarks, i.e., we use the same fermion discretizations for the sea and valence quarks. The two ensembles have different types of fermion discretizations: one is the RBC/UKQCD lattices with domain-wall fermions at $a=0.084 \mathrm{fm}$, and the other is the JLab/William\&Mary lattices with clover fermions at $a=0.114 \mathrm{fm}$. Both are at approximately the same pion mass, close to $300 \mathrm{MeV}$. By comparing results on those two ensembles, we can see the dependence of the TMD observables on discretization effects, including chiral symmetry breaking, and scaling violations. When we define the TMD ratio observables, we assume that soft factors are purely multiplicative, such that they cancel in ratios. We furthermore assume that the ratios also cancel the quark field renormalization factors, so that they are scale invariant. By comparing the two calculations at different scales, we can see to what extent this assumption holds.

\begin{tabular}{c|cccccc}
\hline \hline ID & Fermion Type & Geometry & $a(\mathrm{fm})$ & $m_{\pi}(\mathrm{MeV})$ & \# confs. & \# meas. \\
\hline Clover & Clover & $32^{3} \times 96$ & 0.114 & 317 & 967 & 23208 \\
DWF & Domain-wall & $32^{3} \times 64$ & 0.084 & 297 & 533 & 4264 \\
\hline \hline
\end{tabular}

Table 1: Lattice parameters of the RBC/UKQCD domain-wall ensemble and the JLab/William\&Mary clover ensemble used in this work. $n_{f}=2+1$ for both ensembles.

\section{Results}

In this study, we calculate four TMD observables: (1) generalized Sivers shift, (2) generalized Boer-Mulders shift, (3) transversity $h_{1}$ and (4) generalized worm-gear shift from $g_{1 T}$. 

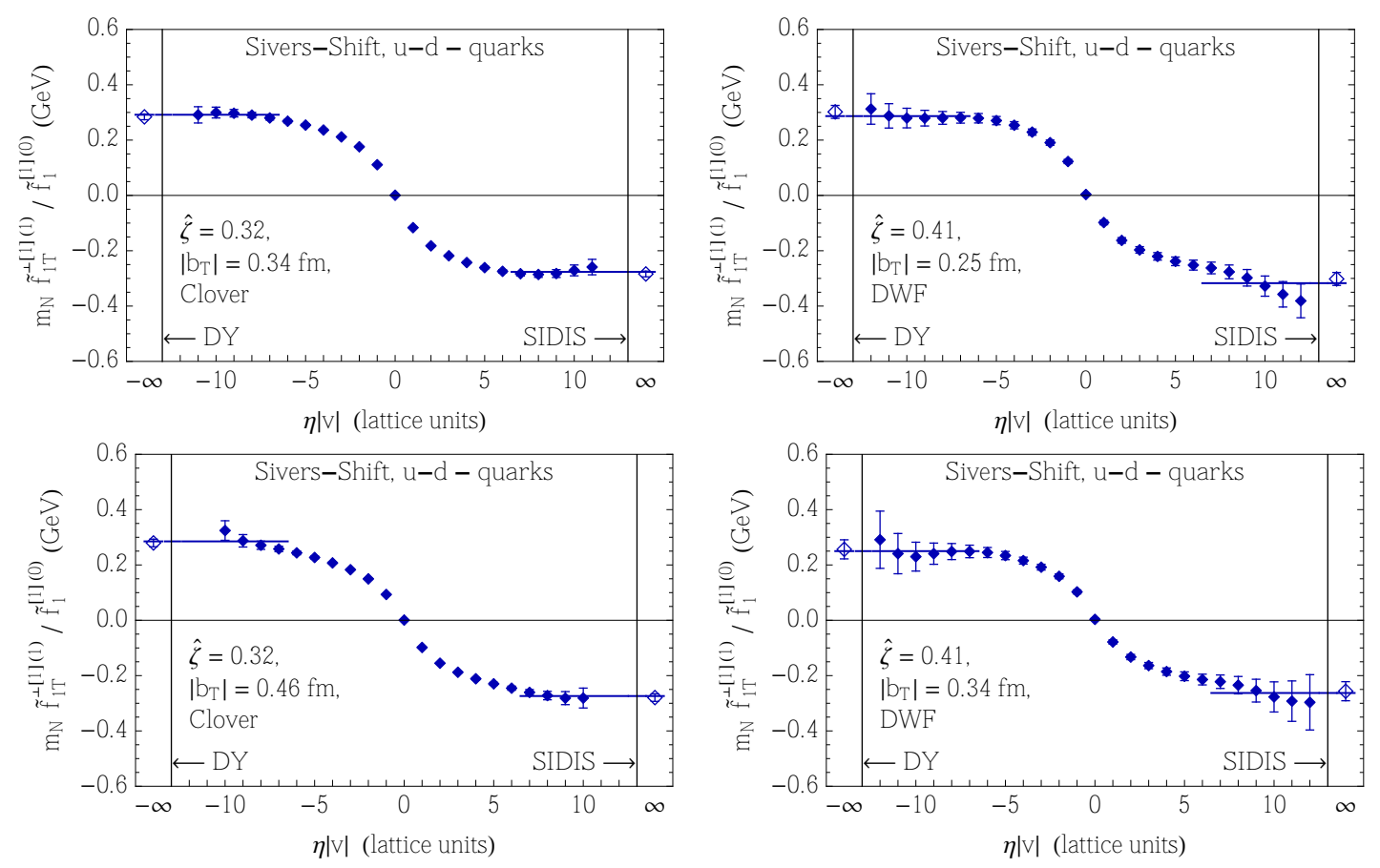

Figure 1: Dependence of the generalized Sivers shift on the staple extent $\eta|v|$ for Clover (left) and DWF (right) ensembles at $\left|\boldsymbol{b}_{\mathrm{T}}\right|=3 a$ (top), and $4 a$ (bottom). Collins-Soper parameter is fixed at the highest value $\hat{\zeta}=0.41$ and 0.32 for DWF and Clover ensembles, respectively.

The generalized Sivers shift addresses the distribution of unpolarized quarks in a transversely polarized proton, and it is defined by $\left\langle\boldsymbol{k}_{y}\right\rangle_{T U}\left(\boldsymbol{b}_{\mathrm{T}}^{2} ; \ldots\right) \equiv m_{N} \tilde{f}_{1 T}^{\perp[1](1)}\left(\boldsymbol{b}_{\mathrm{T}}^{2} ; \ldots\right) / \tilde{f}_{1}^{[1](0)}\left(\boldsymbol{b}_{\mathrm{T}}^{2} ; \ldots\right)$, where $m_{N}$ is the nucleon mass, $f_{1 T}^{\perp}$ is the Sivers function, and $f_{1}$ is the unpolarized function. Here tilde represents the Fourier transformation of a function in the transverse momentum $\boldsymbol{k}_{\mathrm{T}}$-space onto the $\boldsymbol{b}_{\mathrm{T}}$-space, superscript [1] denotes the first Mellin moment in the longitudinal momentum fraction of the quark, $x$, and superscript $(n)$ are defined as $\tilde{f}^{(n)}\left(x, \boldsymbol{b}_{\mathrm{T}}^{2} \ldots\right) \equiv n !\left(-\frac{2}{m_{N}^{2}} \partial_{\boldsymbol{b}_{\mathrm{T}}^{2}}\right)^{n} \tilde{f}\left(x, \boldsymbol{b}_{\mathrm{T}}^{2} ; \ldots\right)$. Detailed descriptions and physical interpretations of the notations are given in Ref. [8].

In order to obtain the generalized Sivers shift in SIDIS or DY process, the staple extent $|\eta||v|$ of the gauge link connecting the two separated quarks needs to be extrapolated to infinity. The $\eta|v|$ dependence of the generalized Sivers shift is shown in Fig. 1. In our setup, DY process is given by the limit $\eta|v| \rightarrow-\infty$, and SIDIS by the limit $\eta|v| \rightarrow \infty$. As predicted in the QCD factorization, DY and SIDIS results show the opposite sign and have the same magnitude. We find that $\eta|v|-$ dependence forms a plateau when $|\eta||v| \geq 7 a$, regardless of the lattice spacing. Hence we obtain the results in DY and SIDIS processes by taking average over $|\eta||v|=7 a \sim \eta_{\max }$, simultaneously in the DY and SIDIS limit, imposing anti-symmetric condition in $\eta|v|$.

Although they form a plateau as they lie on a constant within $1 \sim 2 \sigma$ deviation, we find nonzero slope in some cases, which indicates that there could be remaining systematic error when $|\eta||v|$ is close to $7 a$. As one can see, in addition, the statistical uncertainty increases as the staple extent increases. Hence, if we use weighted average to obtain the asymptotic value, it will be governed by the results at small $|\eta||v|$, where the systematic error is relatively larger than other 

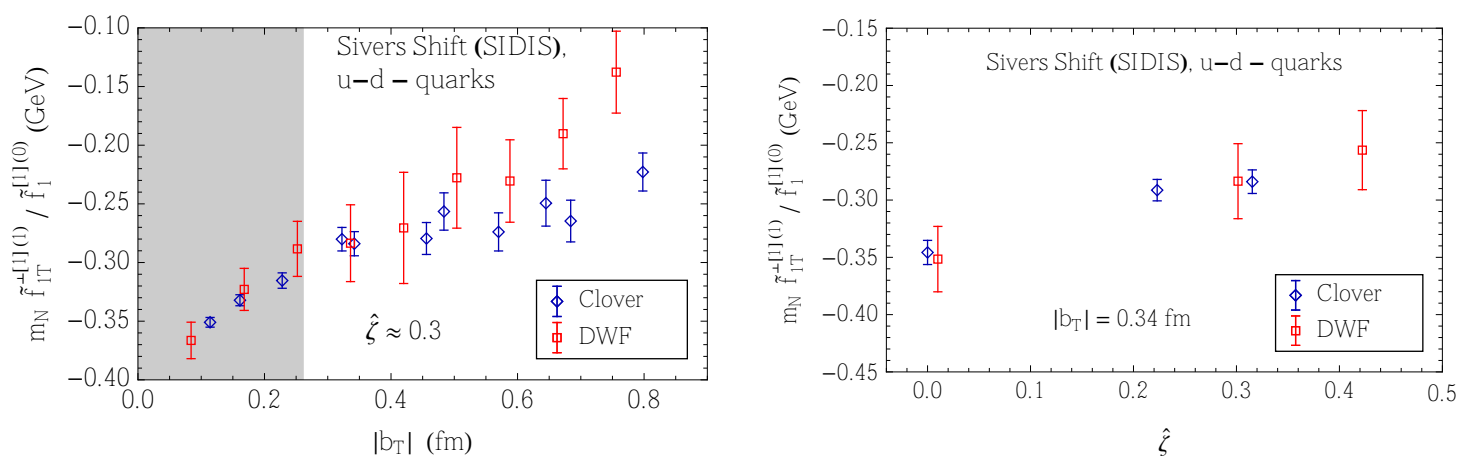

Figure 2: Dependence of the generalized Sivers shift on $\left|\boldsymbol{b}_{\mathrm{T}}\right|$ (left) and $\hat{\zeta}$ (right) for the two different ensembles. In $\left|\boldsymbol{b}_{\mathrm{T}}\right|$-dependence plot $\hat{\zeta}$ is fixed around 0.35 , and in $\hat{\zeta}$-dependence plot $\left|\boldsymbol{b}_{\mathrm{T}}\right|$ is fixed at $0.34 \mathrm{fm}$. When $\left|\boldsymbol{b}_{\mathrm{T}}\right|$ is small, the lattice results are supposed to be contaminated by the lattice cutoff effect. In $\left|\boldsymbol{b}_{\mathrm{T}}\right|$ dependence plot, the shaded area is the small $\left|\boldsymbol{b}_{\mathrm{T}}\right|$ region where $\left|\boldsymbol{b}_{\mathrm{T}}\right| \leq 3 a_{\mathrm{DWF}} \approx 0.25 \mathrm{fm}$.

data points. In order to avoid this problem, we calculate the asymptotic value by using only the mean values of the data points, and calculate the statistical error by using the Jackknife method.

Here we use different $\eta_{\max }$ for different measurements. For Clover, $\eta_{\max }$ is 12 up to $\left|\boldsymbol{b}_{\mathrm{T}}\right|=$ $0.23 \mathrm{fm}, 11$ for $\left|\boldsymbol{b}_{\mathrm{T}}\right|=0.34 \mathrm{fm}, 10$ for $\left|\boldsymbol{b}_{\mathrm{T}}\right|=0.45 \mathrm{fm}$, and so on. For DWF, $\eta_{\max }$ is 12 up to $\left|\boldsymbol{b}_{\mathrm{T}}\right|=0.42 \mathrm{fm}, 10$ for $\left|\boldsymbol{b}_{\mathrm{T}}\right|=0.50 \mathrm{fm}, 9$ for $\left|\boldsymbol{b}_{\mathrm{T}}\right|=0.59 \mathrm{fm}$, and so on. Therefore, the results at large $\left|\boldsymbol{b}_{\mathrm{T}}\right|$ region are obtained only from a few data points $|\eta||v|$ close to $7 a$. Hence, they have smaller statistical error than those at small $\left|\boldsymbol{b}_{\mathrm{T}}\right|$ region, and they may have unresolved systematic error because there are too small number of data points to confirm if we are in an asymptotic region that forms plateau or not.

Fig. 2 shows the dependence of the generalized Sivers shift on $\left|\boldsymbol{b}_{\mathrm{T}}\right|$ (left) and $\hat{\zeta}$ (right) for the two different ensembles. When we define the generalized Sivers shift by the ratio, we assume that the two quarks in the non-local quark bilinear operators are separated far enough so that renormalization constant is independent of the gamma structure of the operator. This assumption is correct only when $\left|\boldsymbol{b}_{\mathrm{T}}\right|$ is large enough. Furthermore, when $\left|\boldsymbol{b}_{\mathrm{T}}\right|$ is small, the lattice results are contaminated by the lattice cutoff effect. In $\left|\boldsymbol{b}_{\mathrm{T}}\right|$-dependence plot, the shaded area represents the small $\left|\boldsymbol{b}_{\mathrm{T}}\right|$ region where $\left|\boldsymbol{b}_{\mathrm{T}}\right| \leq 3 a_{\mathrm{DWF}} \approx 0.25 \mathrm{fm}$. Here the minimum separation $3 a_{\mathrm{DWF}}$ is chosen so that Clover and DWF ensembles of different fermion discretizations and cutoff scales give the consistent results for all the $\left|\boldsymbol{b}_{\mathrm{T}}\right|$-dependences of the observables given in Figs. 2, 3, and 4.

The right-hand side plot on Fig. 2 shows the Collins-Soper evolution parameter $\hat{\zeta}$-dependence of the generalized Sivers shift at fixed $\left|\boldsymbol{b}_{\mathrm{T}}\right|=0.34 \mathrm{fm}$. The $\hat{\boldsymbol{\zeta}}$ is a scale parameter introduced to avoid the rapidity divergences by taking the Wilson line off the light-cone to the space-like region. The light-cone limit is $\hat{\zeta} \rightarrow \infty$, and TMDs with finite $\hat{\zeta}$ can be evolved to the light-cone perturbatively. In order to make the perturbative evolution reliable, however, $\hat{\zeta}$ needs to be large enough so that $\zeta=2 m_{N} \hat{\zeta} \gg \Lambda_{\mathrm{QCD}}$. In this study, we calculate only up to $\hat{\zeta}=0.41$, which is too small to use the perturbativative evolution. The major problem of the lattice QCD calculation at large $\hat{\zeta}$ is the statistical uncertainty; larger $\hat{\zeta}$ calculation requires larger proton momenta, which introduces large statistical error [8].

Another T-odd TMD we calculate is the generalized Boer-Mulders shift, which is defined 

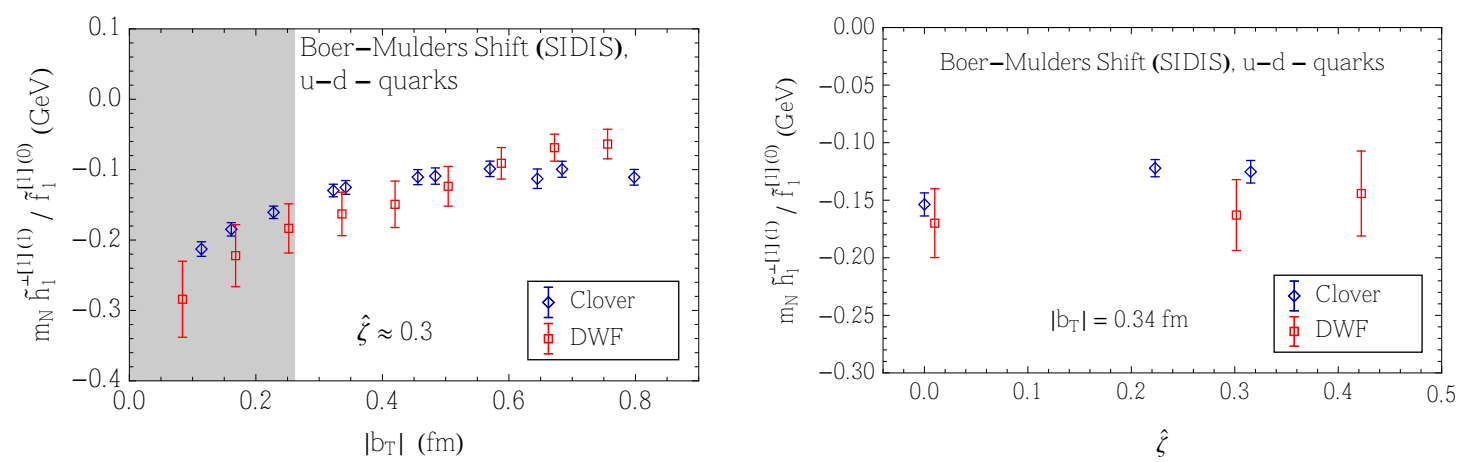

Figure 3: Dependence of the generalized Boer-Mulders shift on $\left|\boldsymbol{b}_{\mathrm{T}}\right|$ (left) and $\hat{\zeta}$ (right) for the two different ensembles. Notations and other parameters are the same as those in Fig. 2.
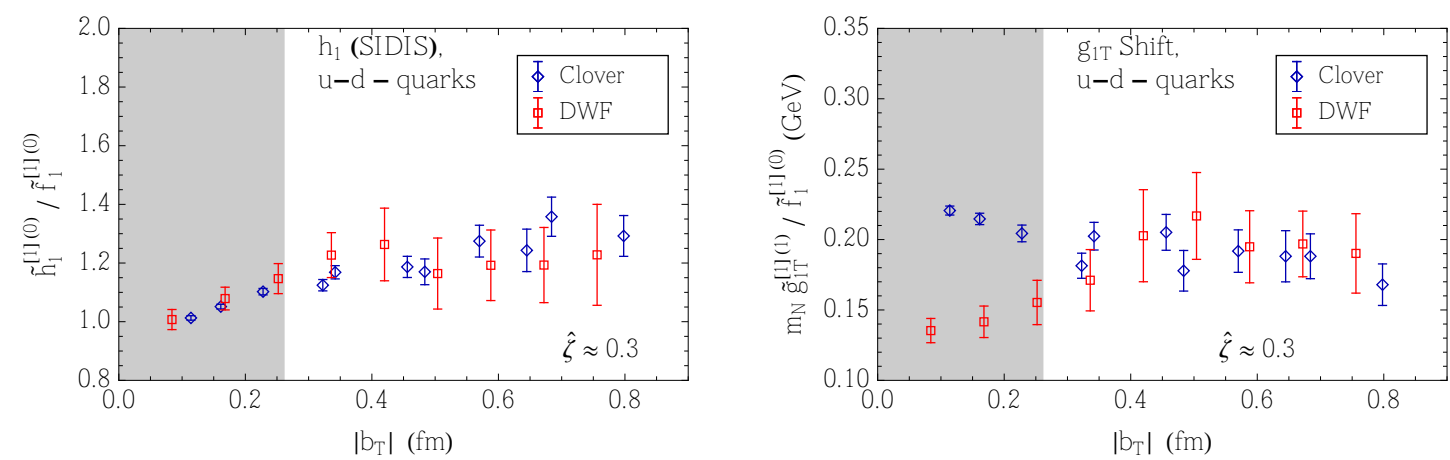

Figure 4: Dependence of the transversity ratio $\tilde{h}_{1}^{[1](0)} / \tilde{f}_{1}^{[1](0)}$ (left) and the generalized $g_{1 T}$ (right) on $\left|\boldsymbol{b}_{\mathrm{T}}\right|$ for the two different ensembles. Notations and other parameters are the same as those in Fig. 2.

by $\left\langle\boldsymbol{k}_{y}\right\rangle_{U T}\left(\boldsymbol{b}_{\mathrm{T}}^{2} ; \ldots\right) \equiv m_{N} \tilde{h}_{1}^{\perp[1](1)}\left(\boldsymbol{b}_{\mathrm{T}}^{2} ; \ldots\right) / \tilde{f}_{1}^{[1](0)}\left(\boldsymbol{b}_{\mathrm{T}}^{2} ; \ldots\right)$, which describes the distribution of transversely polarized quarks in an unpolarized proton. Here $h_{1}^{\perp}$ is the Boer-Mulders function.

The dependence of the Boer-Mulders shift on $\left|\boldsymbol{b}_{\mathrm{T}}\right|$ and $\hat{\boldsymbol{\zeta}}$ for two different ensembles is shown in Fig. 3. We find that the results on the two different Clover and DWF ensembles are consistent within their statistical uncertainty. In the generalized Boer-Mulders shift case, $\hat{\zeta}$ dependence has been studied up to $\hat{\zeta}=2.03$ with pions in Ref. [12], exploiting the fact that signal-to-noise ratio is much larger in pions than in protons. We expect similar scaling behavior in the proton, but it is not clearly shown by this study because calculation extends only up to $\hat{\zeta}=0.41$.

For the rest of this section, we briefly describe the results of the T-even TMDs: the transversity $h_{1}$ and the generalized worm-gear shift $g_{1 T}$. Unlike the T-odd TMDs, such as the Sivers and BoerMulders distributions, T-even TMDs are predicted to be process independent, i.e., same in DY and SIDIS processes. Hence they have been studied with lattice QCD by using the straight Wilson line $[13,7]$, and it has been shown that the difference between straight gauge link and stapleshaped gauge link is small for these T-even TMDs [8]. In this study, we reconfirm this argument for different lattice discretizations and lighter pion masses. We found that most of the results from the two lattice discretizations are consistent with each other, except in the small $\left|\boldsymbol{b}_{\mathrm{T}}\right|$ region of the generalized worm gear shift $g_{1 T}$. In Fig. 4, we show the $\left|\boldsymbol{b}_{\mathrm{T}}\right|$ dependence of the two Teven TMDs. As one can see, the Clover and DWF results of $g_{1 T}$ shift are different when $\left|\boldsymbol{b}_{\mathrm{T}}\right| \leq$ 


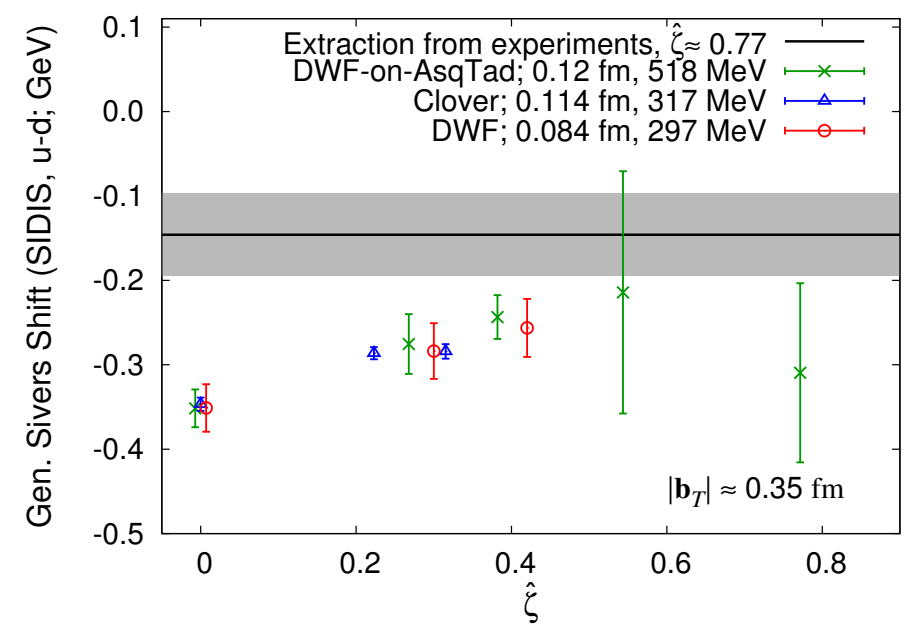

Figure 5: Experimental estimate and lattice QCD calculations of the generalized Sivers shift in SIDIS limit for different Collins-Soper parameter values $\hat{\zeta}$ with various lattice setups. The results of DWF-on-Asqtad are obtained from the previous study of Ref. [8]. The extraction from the experiments has been done at $\hat{\zeta} \approx 0.77$.

$0.25 \mathrm{fm}$. However, we expect large systematic uncertainties regarding the lattice regularization and renormalization when $\left|\boldsymbol{b}_{\mathrm{T}}\right|$ is small, and the results from different discretizations may differ in the region.

\section{Comparison with Phenomenological Estimate and Conclusion}

In this study, we compared the lattice QCD calculation from two different discretizations: $a=0.114 \mathrm{fm}$ Clover vs. $a=0.084 \mathrm{fm} \mathrm{DWF}$ at $m_{\pi} \approx 300 \mathrm{MeV}$. We found that the results of the Clover and DWF ensembles are consistent with each other in the region where lattice artifacts are expected to be small.

In Fig. 5, we compare the current lattice QCD calculations of the generalized Sivers shift with the experimental estimate. The generalized Sivers shift is defined by the ratio of the Sivers function and the unpolarized function. In order to calculate the experimental estimate of the generalized Sivers shift, we use the Sivers shift results of HERMES, COMPASS and Jefferson Lab experiments extracted in Ref. [14]. In the comparison plot, it is clearly shown that the three lattice ensembles with different pion mass and different discretization give consistent results. One also can find that the discrepancy between the central values of phenomenological value and lattice results becomes smaller as $\hat{\zeta}$ increases. Here the $\hat{\zeta}$ is a scale introduced to regulate the rapidity divergences, and the factorization works only when $\hat{\zeta}$ is large enough so that it lies in the perturbative regime. The extraction from the experiments has been done at $\hat{\zeta} \approx 0.77$, which corresponds to $\zeta \approx 1.5 \mathrm{GeV}$, while precise lattice results are obtained at $\hat{\zeta} \leq 0.41$, and the lattice data points at $\hat{\zeta}>0.41$ have huge uncertainty. Going by the behavior we see in the pion study [12], we expect that extrapolating the lattice results to $\hat{\zeta} \rightarrow 0.77$ brings the lattice result close to the extracted value from the experiments. 


\section{Acknowledgments}

Computations were performed using resources provided by the U.S. DOE Office of Science through the National Energy Research Scientific Computing Center (NERSC), a DOE Office of Science User Facility, under Contract No. DE-AC02-05CH11231, as well as through facilities of the USQCD Collaboration, employing the Chroma software suite [15]. The RBC/UKQCD collaboration is gratefully acknowledged for providing gauge configurations analyzed in this work, as are K. Orginos (supported by DOE grant DE-FG02-04ER41302) and the Jefferson Lab lattice group (supported by DOE grant DE-AC05-06OR23177, under which Jefferson Science Associates, LLC, operates Jefferson Laboratory). Support by the Heisenberg-Fellowship program of the DFG (P.H.), the PRISMA Cluster of Excellence at the University of Mainz (J.G.), and the RIKEN Foreign Postdoctoral Researcher Program (BNL) as well as the Nathan Isgur Fellowship (JLab) (S.S.) is acknowledged. This work was furthermore supported by the U.S. DOE and the Office of Nuclear Physics through grants DE-FG02-96ER40965 (M.E.), DE-SC0011090 (J.N.) and DE-FC0206ER41444 (A.P.). R.G., T.B. and B.Y. are supported by DOE grant DE-KA-1401020 and the LDRD program at LANL.

\section{References}

[1] X. d. Ji, J. P. Ma and F. Yuan, Phys. Lett. B 597, 299 (2004) [hep-ph/0405085].

[2] X. d. Ji, J. p. Ma and F. Yuan, Phys. Rev. D 71, 034005 (2005) [hep-ph/0404183].

[3] S. M. Aybat and T. C. Rogers, Phys. Rev. D 83, 114042 (2011) [arXiv:1101.5057 [hep-ph]].

[4] A. Airapetian et al. [HERMES Collaboration], Phys. Rev. Lett. 103, 152002 (2009) [arXiv:0906.3918].

[5] C. Adolph et al. [COMPASS Collaboration], Phys. Lett. B 717, 383 (2012) [arXiv:1205.5122.

[6] C. Van Hulse [HERMES Collaboration], EPJ Web Conf. 85, 02020 (2015).

[7] B. U. Musch, P. Hägler, J. W. Negele and A. Schäfer, Phys. Rev. D 83, 094507 (2011) [arXiv:1011.1213 [hep-lat]].

[8] B. U. Musch, P. Hägler, M. Engelhardt, J. W. Negele and A. Schäfer, Phys. Rev. D 85, 094510 (2012) [arXiv:1111.4249 [hep-lat]].

[9] J. C. Collins and D. E. Soper, Nucl. Phys. B 194, 445 (1982).

[10] J. C. Collins and D. E. Soper, Nucl. Phys. B 193, 381 (1981) [Nucl. Phys. B 213, 545 (1983)].

[11] S. M. Aybat, J. C. Collins, J. W. Qiu and T. C. Rogers, Phys. Rev. D 85, 034043 (2012) [arXiv:1110.6428 [hep-ph]].

[12] M. Engelhardt, B. Musch, P. Hägler, J. Negele and A. Schäfer, PoS LATTICE 2013, 284 (2014) [arXiv:1310.8335 [hep-lat]].

[13] P. Hägler, B. U. Musch, J. W. Negele and A. Schäfer, Europhys. Lett. 88, 61001 (2009) [arXiv:0908.1283 [hep-lat]].

[14] M. G. Echevarria, A. Idilbi, Z. B. Kang and I. Vitev, Phys. Rev. D 89, 074013 (2014) [arXiv:1401.5078 [hep-ph]].

[15] R. G. Edwards et al. [SciDAC and LHPC and UKQCD Collaborations], Nucl. Phys. Proc. Suppl. 140, 832 (2005) [hep-lat/0409003]. 